\title{
Changes of the Molecular Structure in Organic Thin Film Transistors during Operation
}

Fabiola Liscio*, ${ }^{1}$ Laura Ferlauto, ${ }^{1}$ Micaela Matta, ${ }^{2}$ Raphael Pfattner ${ }^{3}$, Mauro Murgia ${ }^{4}$, Concepció Rovira $^{3}$, Marta Mas-Torrent ${ }^{3}$, Francesco Zerbetto, ${ }^{2}$ Silvia Milita ${ }^{1}$ and Fabio Biscarini ${ }^{5}$

${ }^{1}$ CNR - Istituto per la Microelettronica e Microsistemi (IMM), I-40129 Bologna, Italy

${ }^{2}$ Dip. di Chimica “G. Ciamician”, Università di Bologna, 40126 Bologna, Italy

${ }^{3}$ Institut de Ciencia de Materials de Barcelona (ICMAB-CSIC) and Networking Research Center on Bioengineering, Biomaterials and Nanomedicine (CIBER-BBN), 08193 Bellaterra, Spain

${ }^{4}$ CNR - Istituto per lo Studio dei Materiali Nanostrutturati (ISMN), I-40129 Bologna, Italy

${ }^{5}$ Dip. Scienze della Vita, Università di Modena e Reggio Emilia, 41125 Modena, Italy

Corresponding author: liscio@bo.imm.cnr.it

KEYWORDS: real time X-ray diffraction; lattice strain; organic field-effect transistors; bias stress; pentacene.

\begin{abstract}
Thin films of organic semiconductors have been widely studied at different length scales for improving the electrical response of devices based on them. Hitherto, a lot of knowledge has been gained about how molecular packing, morphology, grain boundaries, and defects affect the charge transport in organic thin film transistors. However, little is known about the impact of an electric field on the organic semiconductor microstructure and the consequent effect on the device performances. To fill this gap, we investigated the evolution of the structure of pentacene thin film transistors during device operation by in situ real time X-ray diffraction measurements and theoretical calculations. We observed for the first time the occurrence of a reversible structural strain taking place during the bias application mainly due to reorientation at the terrace edges of monolayer islands under the effect of electrical field. Strain exhibits the same trend of the threshold voltage hinting to the existence of a direct correlation between the phenomenon of bias stress and the structural modification.
\end{abstract}




\section{INTRODUCTION}

Understanding the molecular organization at multiple length scales is crucial for unravelling the physics of Organic Field Effect Transistors (OFETs). ${ }^{1-5}$ The sub-nm scale is the typical distance of intermolecular and $\pi$-stacking interactions, which determine the hopping rate of charge carriers and polarization effects; the 1-10 nm scale is the effective Debye length of the organic semiconductor that corresponds to the thickness of the accumulation layer; the longitudinal electrostatic potential drops across charge injection interfaces and domain boundaries on the $10 \mathrm{~nm}$ scale; the 100-1000 $\mathrm{nm}$ is the lateral length scale of semiconductor domains; tens of micrometers is the channel length where charge carriers move.

The observation that field effect mobility, $\mu$, is usually larger in highly crystalline and textured organic semiconductor film ${ }^{6-8}$ has prompted the design of materials for OFET able to yield tight molecular packing and a high degree of orientation. Crystalline order, although necessary, does not guarantee that the resulting semiconductor thin film possesses the "desired" order. Thin film growth is an out of equilibrium process whose outcome depends on the interplay of competing kinetic processes. In the case of high-vacuum sublimed semiconductor molecules, control of molecular order is achieved by changing the substrate temperature, ${ }^{2}$ the deposition rate or by applying an electric field. ${ }^{9}$ Thermal and solvent annealing are commonly used for increasing molecular order $e x$ post. $^{10,11}$ In the case of soluble organic semiconductors, other approaches can be exploited to govern molecular order: low dimensional crystallisation upon confinement induced by an external agency, ${ }^{12,13}$ chemical modification of the dielectric interface, ${ }^{14,15}$ introduction of lattice strain by using solution-shearing method. ${ }^{16}$ Conformational degrees of freedom designed in the chemical structure ${ }^{17}$ can be functional to lowering the barriers for reorganization upon annealing. ${ }^{2}$

The correlations established between molecular ordering and OFET parameters are empirical since most of the measurements are carried out on thin films grown on smooth substrates. In real devices thin films are grown or deposited on test patterns that are chemically and topographically inhomogeneous. In addition, the microstructure of organic semiconductors in a thin film transistor cannot be simply ascribed to the ideal bulk structure of the most stable polymorph. In pentacene, sexithienyl and perylene derivatives ${ }^{2,18,19}$ molecular order changes across the film thickness. The first monolayers, in contact with the gate dielectrics (in the bottom-gate architecture) are reminiscent of the thin film polymorph, the upper layers evolve towards one of the bulk phase polymorphs. This thickness-dependent orientational transition is often, albeit not always, continuous. It has been reported the coexistence of molecules with planar orientation at island edges and domain boundaries with standing molecules. ${ }^{20}$ Whether or not the planar molecules reorient and 
are incorporated at later stages into the domains is still a matter of debate. In-plane structural inhomogeneity induced by the dielectric surface has also been reported. ${ }^{21}$

The electric field in OFET is central for charge generation and charge mobility according to Frenkel-Poole theory. ${ }^{22}$ The longitudinal electrostatic potential drops rapidly at grain boundaries thus generating a step-wise electric field profile across the OFET channel ${ }^{23}$ with practically no-field within the domains and large field across boundaries. ${ }^{24}$ The molecular packing motif at the grain boundaries affects charge transport across adjacent crystalline grains, ${ }^{25}$ evidencing that charge transport follows percolation pathways along grain boundaries, which depend on the orientation of adjacent crystalline domains. ${ }^{26}$

The coupling of the transversal electric field with the charge carriers to produce to the so-called bias stress demands to be fully understood. The bias stress has been ascribed mainly to migration and reversible trapping of cations, specifically protons, at the dielectric/semiconductor interface. ${ }^{27}$ In the vacuum, bias stress still exists, suggesting the existence of an intrinsic component to this phenomenon. Although the transversal field may not be strong, being at its maximum on the order of $10^{5} \mathrm{volt} / \mathrm{cm}$, yet it may couple to the charge carriers through polarization, leading to local structural changes and energy lowering of the charged state. Diffuse scattering from electron diffraction has recently revealed distortion of the molecular structure due to the lattice vibrational modes, ${ }^{28}$ which are related with the degree of charge carrier delocalization. ${ }^{29}$ As unique structural investigation performed during device operation, low-frequency phonon Raman spectroscopy revealed a structural modification induced by the source to drain field where the intermolecular distance was reduced after long-term operations. ${ }^{30}$

In this work we present a direct measurement of the structural evolution of pentacene thin film in OFET observed during the application of gate bias, $\mathrm{V}_{\mathrm{GS}}$, which provides a transversal electric field. This investigation was performed by means of X-Ray Diffraction (XRD) measurements, both in specular and Grazing Incidence (GIXRD) geometries, in real time during the application of drainsource $\mathrm{V}_{\mathrm{DS}}$ and gate $\mathrm{V}_{\mathrm{GS}}$ voltages. Selected Bragg reflections characteristic of the lattice planes perpendicular and parallel to the dielectric surface were monitored during OFET operation. The effect of $\mathrm{V}_{\mathrm{GS}}$ on the pentacene crystal structure was simulated by means of Molecular Dynamics (MD) and Density Functional Theory (DFT). The interplay between the calculations and the experiments provides an understanding of the structural evolution of operating transistors. The transversal field induces a slight change of orientation of the molecules within the domains. Instead, and more relevantly, for a small fraction of molecules located either at the grain boundaries or near them, the re-orientation is substantial and leads to a significant energy lowering. This implies that 
the re-oriented molecules at grain boundaries might act as shallow traps for charge carriers, hinting to a new explanation for origin of bias stress in OFET devices

\section{RESULTS AND DISCUSSION}

The OFET is schematically depicted in Figure 1. It is based on six-to-ten monolayer (ML) thick pentacene thin films (9-15 nm nominal thickness). Channel length and width are $300 \mu \mathrm{m}$ and 10 $\mathrm{mm}$, respectively. This channel length is larger than typical devices (tens of micrometers). The layout with linear electrodes allows the X-ray beam to probe the channel region during device operation while avoiding the Au electrodes.

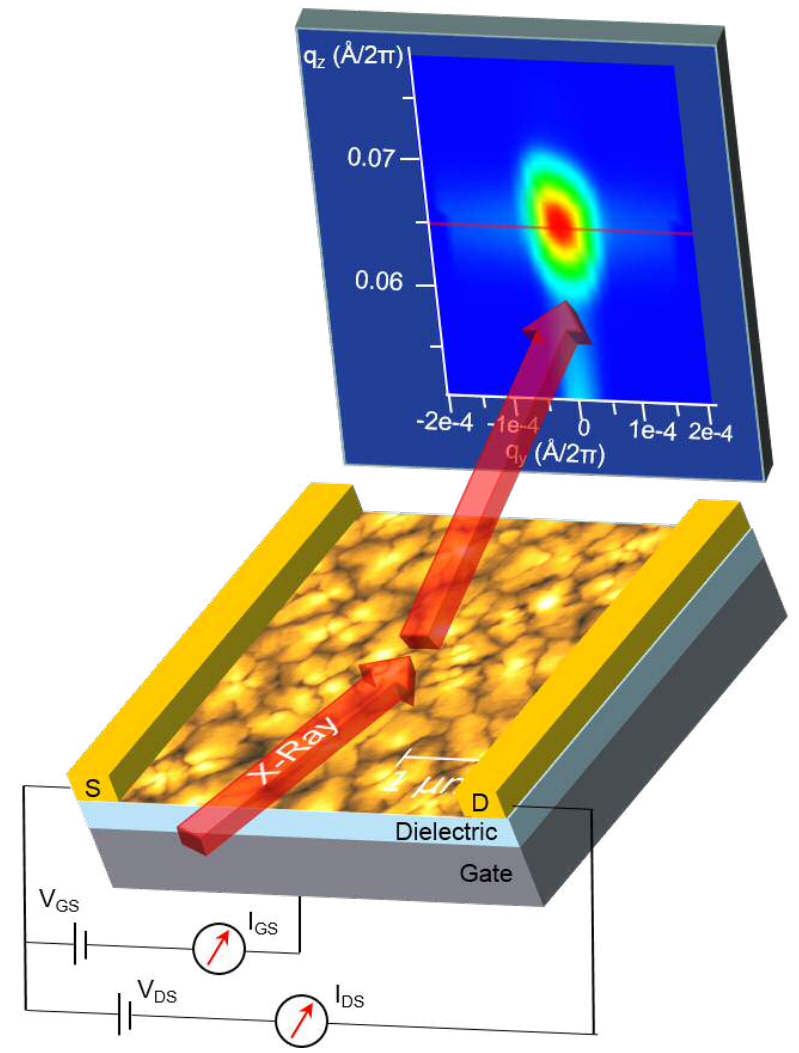

Figure 1. Schematics of the pentacene OFET. AFM image of the pentacene thin film and Reciprocal Space Map (RSM) around the (001) reflection of a 10ML pentacene film.

Figure 1 shows an example of the RSM collected around the (001) reflection and AFM image of a pristine 10MLs pentacene film. The RSM indicates that pentacene molecules crystallize in "thin film" phase as deduced from the peak position, i.e. $\mathrm{d}_{001}=1 \mathrm{ML}=15.5 \AA$. The Bragg peak width reveals a highly textured film characterized by a rough surface. AFM images yield $1.7 \mathrm{~nm}$ saturated root mean square roughness . 
The time dependent evolution of the pentacene structure during the OFET operation was studied by monitoring the X-ray beams diffracted by lattice planes parallel (001) and perpendicular (110) to the film surface. Complete description of the experimental method is reported in the Supporting Information. Preliminary measurements concerned the control of the exposure time to the highly collimated X-ray synchrotron beam generating photoelectrons which may affect the OFET response on one hand, and consequently the parameters extracted, and, on the other hand cause radiation damage to the organic film. ${ }^{31-33}$

By applying $\mathrm{V}_{\mathrm{DS}}=\mathrm{V}_{\mathrm{GS}}=-20 \mathrm{~V}$, the source-drain current $\left(\mathrm{I}_{\mathrm{DS}}\right)$ and the source-gate current $\left(\mathrm{I}_{\mathrm{GS}}\right)$ were continuously measured (Figure 2a) while X-ray measurements around the 001 reflection were carried out every 40 minutes $(\theta / 2 \theta$ and $\omega$ scan, RC, reported in Figure S5) to monitor the evolution of stacking layer periodicity. The occurrence of the bias stress during the device operation is evidenced by the characteristic decrease of $\left|I_{\mathrm{DS}}\right|$, compensated by the photocurrent generated by Xray irradiation (inset in Figure 2a). The (001) RC continuously shifts towards smaller angles, by $\Delta \omega=0.002^{\circ}$, and the threshold voltage $\mathrm{V}_{\mathrm{TH}}$, extracted from the transfer curves, decreases from $0 \mathrm{~V}$ to $-1.7 \mathrm{~V}$ (see red points in Figure 2b,c). These structural and electrical variations are reversible, i.e, initial values are recovered when the bias is inverted $\left(V_{G S}=20 \mathrm{~V}\right.$ and $V_{D S}=-1 \mathrm{~V}$, black dots in Figure 2b,c) or switched off (green dots in Figure $\mathbf{2 b , c}$ ), and they re-appear when negative gate bias is re-applied $\left(\mathrm{V}_{\mathrm{GS}}=\mathrm{V}_{\mathrm{DS}}=-30 \mathrm{~V}\right.$, blue dots in Figure $\left.\mathbf{2 b , c}\right)$.
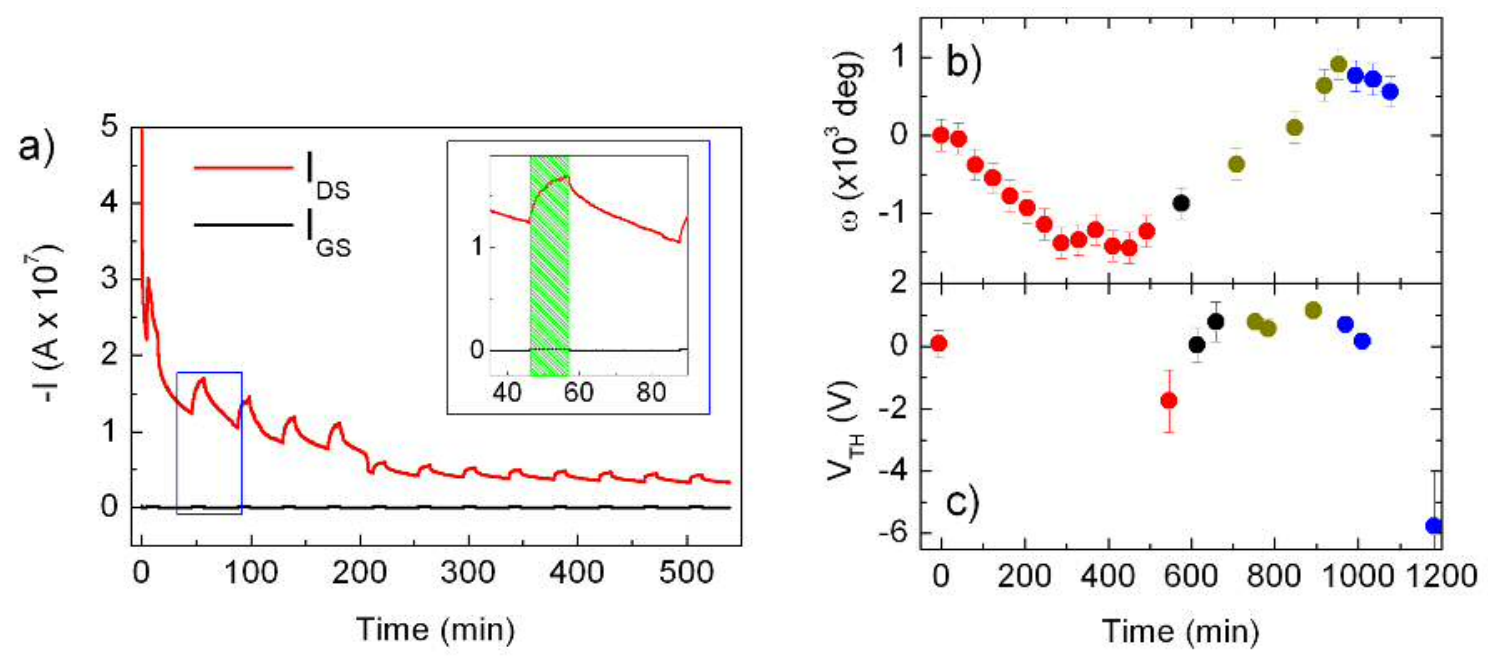

Figure 2. $\mathrm{V}_{\mathrm{GS}}=\mathrm{V}_{\mathrm{DS}}=-20 \mathrm{~V}$ : (a) $\mathrm{I}_{\mathrm{DS}}$ and $\mathrm{I}_{\mathrm{GS}} v$ s time. The green box in the inset indicates the time when an XRD scan is performed. (b) $\mathrm{RC}(001)$ peak position and c) $\mathrm{V}_{\mathrm{TH}}$ values extracted from transfer curves in time, when $\mathrm{V}_{\mathrm{GS}}=\mathrm{V}_{\mathrm{DS}}=-20 \mathrm{~V}$ (red dots), $\mathrm{V}_{\mathrm{GS}}=20 \mathrm{~V}$ and $\mathrm{V}_{\mathrm{DS}}=-1 \mathrm{~V}$ (black dots), $\mathrm{V}_{\mathrm{GS}}=\mathrm{V}_{\mathrm{DS}}=0 \mathrm{~V}$ (green dots), $\mathrm{V}_{\mathrm{GS}}=\mathrm{V}_{\mathrm{DS}}=-30 \mathrm{~V}$ (blue dots). Absolute error of $\mathrm{V}_{\mathrm{TH}}$ calculated with $95 \%$ of confidence. 
The RC shift to smaller angles can be ascribed to a lattice expansion along the film thickness (i.e. molecular layer thickness) of $0.016 \AA$, due to a molecular tilt of $2.3 \mathrm{mrad}$ toward the surface normal. Indeed, this molecular tilt is directly revealed by the shift of the (001) peak recorded with a 2D detector after $1 \mathrm{~h}$ of $\mathrm{V}_{\mathrm{G}}=\mathrm{V}_{\mathrm{DS}}=-30 \mathrm{~V}$ (Figure 3a,b,c).
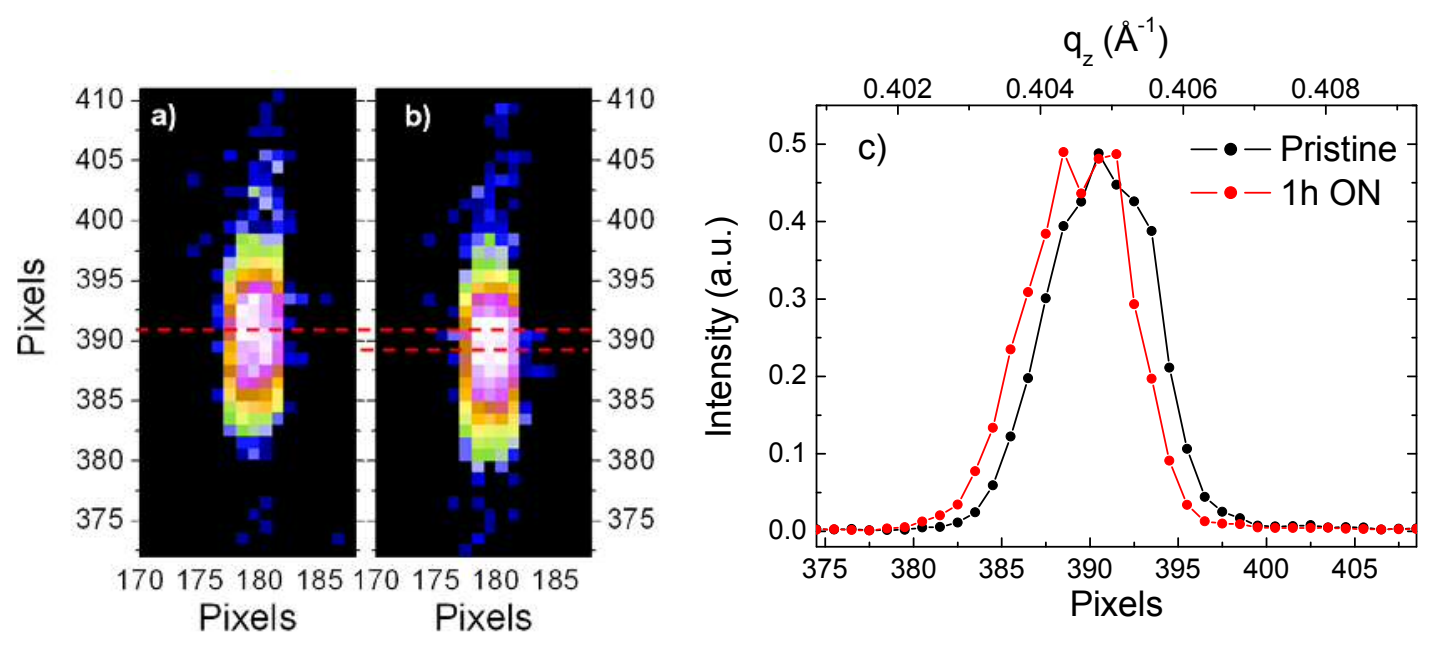

Figure 3. 2D detector images collected at (001) Bragg condition before (a) and after (b) $1 \mathrm{~h}$ of $\mathrm{V}_{\mathrm{GS}}=$ $\mathrm{V}_{\mathrm{DS}}=-30 \mathrm{~V}$. c) Comparison between vertical slices of the images along the specular direction.

Interestingly the relationship between the vertical electric field effect and the pentacene structural vertical stress is confirmed by the occurrence of the RC shift when a significant leakage current appears, following the same trend observed when the transistor is switched off (Figure S8). This behaviour can be construed as due to the fact that when the leakage current increases the effective electric field built up in the transistor channel decreases.

An additional evidence of the field effect on the film structure is pointed out by the dependence of the RCs shift on film thickness. When the film thickness is comparable to the effective Debye length, i.e. $4 \mathrm{MLs},{ }^{3}$ after already 11 minutes of $\mathrm{V}_{\mathrm{G}}=\mathrm{V}_{\mathrm{SD}}=-40 \mathrm{~V}$ the RC shifts $0.004^{\circ}$ (Figure S9a). In contrast, when the film thickness is very high (50MLs, Figure S9b) XRD measurements probe the full film depth, within which 45 MLs are not field affected and the RC remains unchanged even after 144 minutes of bias application.

Further information on the pentacene structural changes has been obtained by analysing the evolution of the in-plane structure, i.e. by recording 2D-GIXD images around $\{11\}$ rod, the strongest in-plane reflections, when applying $\mathrm{V}_{\mathrm{GS}}=\mathrm{V}_{\mathrm{DS}}=-40 \mathrm{~V}$. The 2D GIXD images collected before applying biases show an elongated Bragg spot coming from (1-10) and (-1-10) reflections, enhanced at Yoneda position ${ }^{34}$ (i.e. when the exit angle of the scattered beam is close to the critical angle) due to the Vineyard effect ${ }^{35}$ (see Figure $4 \mathbf{a}$ and more details in Figure S4). 
After 120 minutes of OFET bias the rod intensity increases, as clearly observed in the integrated intensity plotted both along the vertical direction (plotted in Figure $4 \mathbf{b}$ and Figure S4a) and along the Yoneda peak (Figure 4c). However, neither a significant shift nor a width change can be detected.

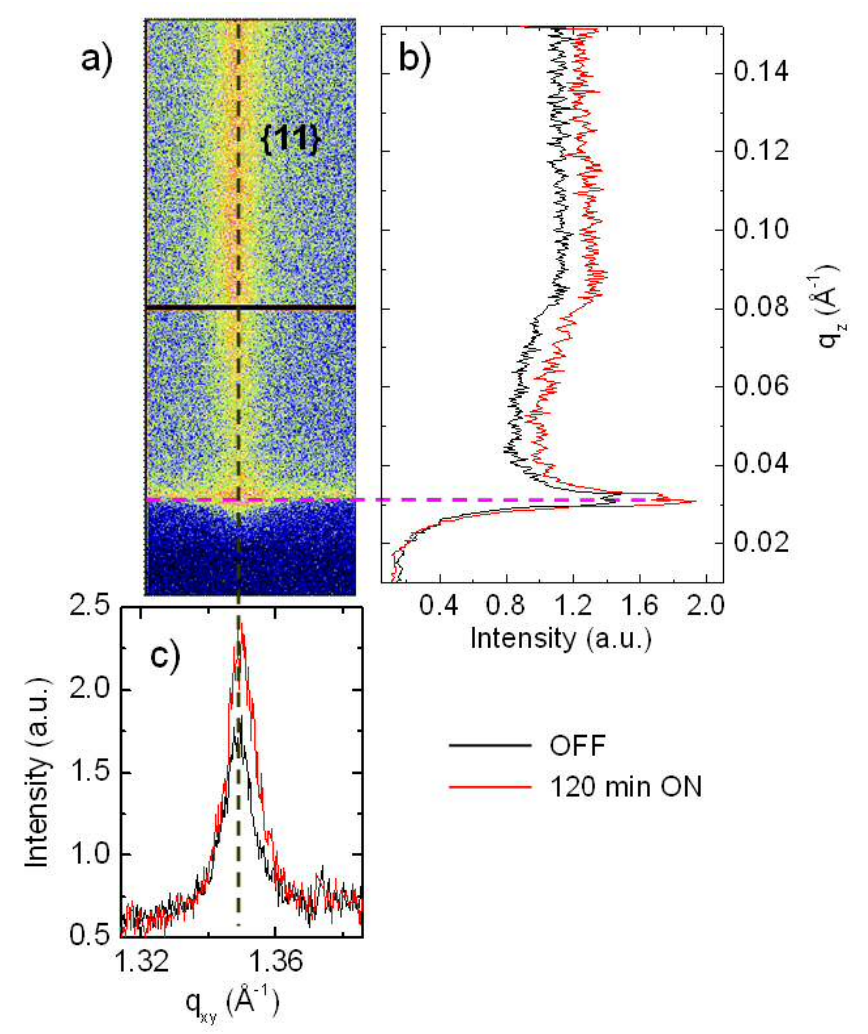

Figure 4. a) $2 \mathrm{DGIXD}$ image collected on pristine $6 \mathrm{MLs}$ pentacene film. Scattering intensity integrated along (b) the rod at $\mathrm{q}_{\mathrm{xy}} \sim 1.35 \AA^{-1}$ and along (c) the Yoneda peak at $\mathrm{q}_{\mathrm{z}} \sim 0.3 \AA^{-1}$ for the images collected before (black line) and after 120 minutes (red line) applying biases.

The in-plane peak evolution can be explained with the enhancement of the crystalline order during OFET operation without any change of the in-plane lattice parameter and the lateral domain coherence. The latter value is $58 \mathrm{~nm}$, as evaluated from the peak width along $\mathrm{q}_{\mathrm{xy}}$ direction, using Scherrer's formula ${ }^{36}$ and taking into account the beam footprint as described in ref ${ }^{37}$.

The contribution to that ordering can be attributed to the healing of point defects inside crystal domains, and to the molecular rearrangement at the grain boundaries and terrace edges. There, molecules can lie planar as demonstrated by AFM and STM measurements, ${ }^{20}$ or adopt a configuration different from the crystal phase. ${ }^{2}$ Because of the looser packing, their energy barriers for re-orientation are smaller with respect to molecules inside the domains. 
To corroborate and understand the experimental findings, computational studies were performed on two systems. The first one consisted in four pentacene molecules in the vacuum forming a tetramer. The DFT calculations, which included dispersion forces, showed that in the absence of electrical field tetramer stabilizes in a structure (named A in Figure 5a), which is more compact and characterized by at lower energy than the structure that can be extracted from the thin film 38,39 ( structure B in Figure 5b). In the presence of an electric field along the long axis (simulating the gate field), structures A and B become equi-energetic.
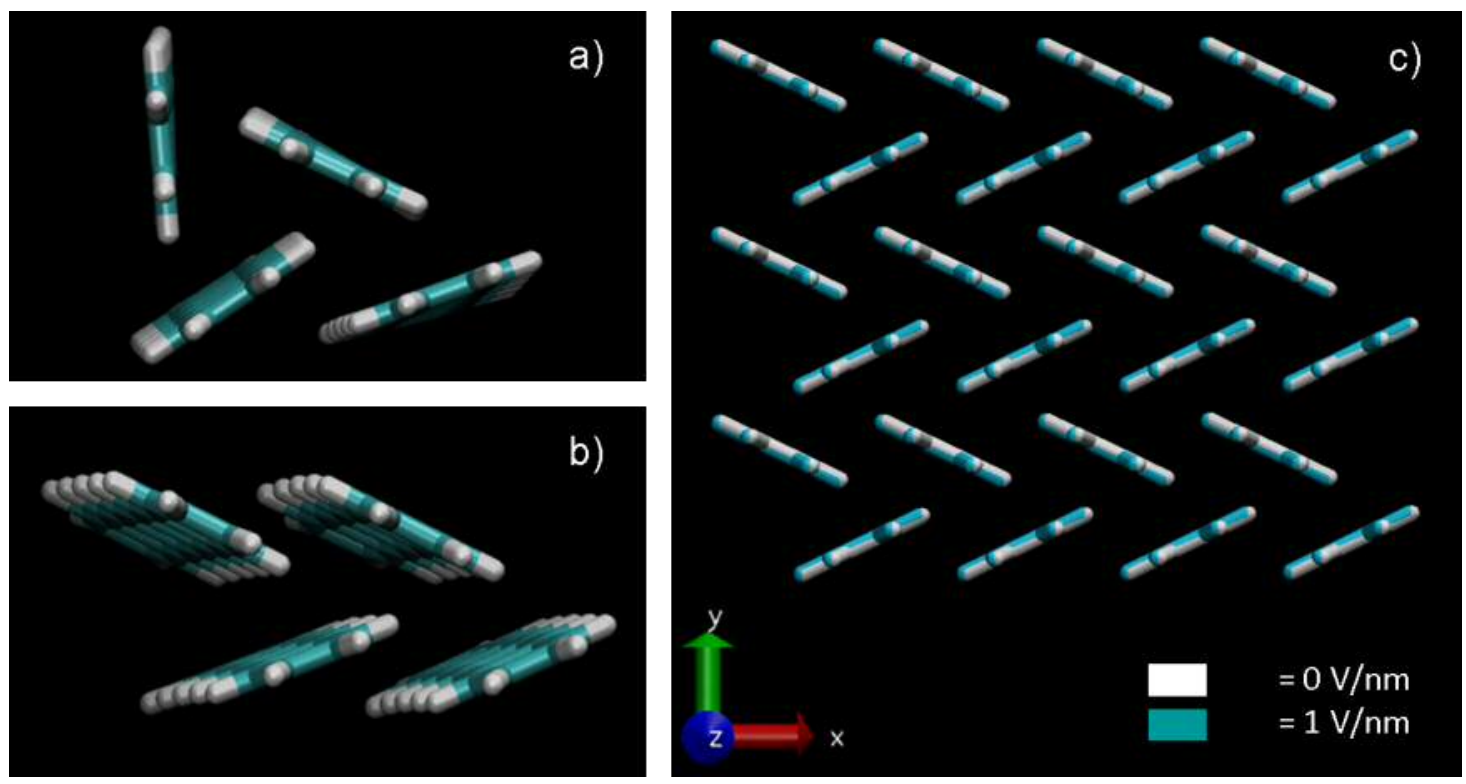

Figure 5. Representation of structure A (a) and structure B (b) of a tetramer in vacuum and the thin film phase. c) Optimization of lattice parameters and atomic coordinates for a supercell made of 24 pentacene molecules (see Table SI2) with an applied electric field along the $z$ direction simulating the field created by the gate voltage. The optimized structures at $0 \mathrm{~V} / \mathrm{nm}$ (white) and $1 \mathrm{~V} / \mathrm{nm}$ (color) are shown.

In the second systems, pentacene molecules were set up in the periodic structure of the thin film phase. ${ }^{38}$ They were located on top of an implicit surface that corresponds to the $\mathrm{SiO}_{2}$ surface in the experimental setup. A full crystal minimization was performed, also using MD calculations. In this case the vertical electrical field slightly affects the crystal structure (see Figure 5c), causing the molecules to align to the vertical axis and reducing the tilt angle from $0.56^{\circ}(0 \mathrm{~V} / \mathrm{nm})$ to $0.32^{\circ}(1$ $\mathrm{V} / \mathrm{nm})$.

By applying a field of $1 \mathrm{~V} / \mathrm{nm}$, molecules tilt only $2 \mathrm{mrad}$ towards the direction normal to the surface, the value is very close to that observed experimentally. In the simulation, the applied field 
is likely to be higher than the real one. The response is almost immediate while it takes several hours to build up experimentally. The simulation and the strain effect response is almost immediate compared to the several hours needed for observing the Bragg peak shift. These discrepancies can be explained as follows. The simulations were limited to $1 \mathrm{ML}$ thick film. XRD measurements probe the full film depth (6 or 10MLs), where the upper molecules are constrained by the upper neighbors potentially restricting their degrees of freedom. Moreover, the simulated electric field is constant, while in the real system each layer screens the upper ones from the transversal electric field, reducing the field effect until the fourth ML, as deduced from the effective Debye length calculated on films grown in the same condition. ${ }^{3}$ These observations also explain the discrepancy between the $\mathrm{V}_{\mathrm{TH}}$ and $\mathrm{RC}$ peak position recovering after applying a positive gate bias (Figure 2bc) ascribing to the different probed scales. $\mathrm{V}_{\mathrm{TH}}$ is evaluated from transfer curves which probe the electrical properties of the very first layers, whereas XRD measurements probe the full film depth.

The tetramer field-induced reorganization can be associated to a scenario where the number of neighbours is lower than in the crystal, for instance, at grain boundaries ${ }^{40}$ and terrace edges. The presence of a field may modify these structures toward the crystalline one, thus explaining the increasing of the in-plane peak intensity as a structural ordering. The process is reversible and cannot be used to order the film. Upon switching off the gate potential, the crystal-like structure is destabilized and the pentacene layer returns disordered. If the gate bias is reversed, a new force, with a different direction is introduced and the net effect is to disorder the film. Due to the small amount of molecules forming the grain boundaries, the crystal domain size remains mostly unaffected by the molecular reorganization justifying why the in-plane Bragg peak width is unchanged, regardless of OFET operation. The change of packing motif at the grain boundaries may perturb the charge hopping between crystal grains ${ }^{25}$ : molecules re-organize towards the molecular packing of the closer crystalline grain, and a spatial gap may be formed between two crystalline grains creating, therefore, charge traps ${ }^{24}$. It follows that this phenomenon may be related with the $\mathrm{V}_{\mathrm{TH}}$ variation, i.e. the bias stress, introducing a new scenario to the comprehension on charge transport: OFET operation does not only depend on the microstructure of the semiconductor film but also on how the microstructure responds to the applied field and modulates the carrier transport during time.

\section{CONCLUSIONS}

Real time XRD measurements performed on few MLs pentacene films grown on a FET device operated at fixed gate have evidenced structural modification during OFET operation. The 
experimental results, coupled with the computational studies, showed that the application of a transversal electrical field $\left(\mathrm{V}_{\mathrm{GS}}\right)$ slightly perturbs the molecular arrangement of crystallites, by inducing a mrad molecular tilt towards the direction of the applied field. Conversely, molecules reorganize at grain boundaries making them as responsible, at least partly, for the $\mathrm{V}_{\mathrm{TH}}$ shift, characteristic of the bias stress phenomenon. The structural modification observed here concerns pentacene that is a polarizable molecule forming highly crystalline and textured crystal domains. These phenomena may be stronger for more polar molecules and/or disordered system (e.g. polymers), opening up new perspectives for the interpretation of charge transport in organic materials.

\section{EXPERIMENTAL METHODS}

Device preparation: pentacene films were grown by high vacuum sublimation at a rate of 0.5 $\mathrm{ML} / \mathrm{min}$ on $\mathrm{SiO}_{2}(200 \mathrm{~nm}) / \mathrm{Si}$ surface at room temperature. Gold electrodes $(40 \mathrm{~nm})$ were evaporated later on the top of it using a shadow mask.

Morphology characterization: topographical AFM characterization was carried out in intermittent contact mode in air environment making use of a Smena, NT-MDT (Moscow, Russia). Data analysis was performed using Gwyddion software.

Structural characterization: RSM of (001) peak was performed using a SmartLab-Rigaku diffractometer equipped with a rotating anode $(\mathrm{Cu} \mathrm{K}, \lambda=1.54056)$. Specular scans and RCs were collected at two synchrotron facilities. At MCX-ELETTRA beamline, the beam wavelength was $1.0332 \AA$ and size was $\mathrm{HxV}=200 \mu \mathrm{m} \times 300 \mu \mathrm{m}$; diffraction peaks were measured by a point detector and the angular acceptance defined by secondary slits before detector was $0.023^{\circ}$ for the specular scans and $0.005^{\circ}$ for the RC measurements. Due to low resolution arising from the Bragg peak broadness related to the small film thickness $\left(\mathrm{FWHM}=0.03 \AA^{-1}\right.$, Figure S5), further specular scans and RCs were collected by a 2D detector at ID01-ESRF beamline with a monochromatic beam of $\lambda=1 \AA$. The 2D images when incident and detector angles are at the Bragg position reveal a partial zone of the RSM around 001 peak that is recorded with improved resolution (Figure S6). Beam size was $\mathrm{HxV}=1.8 \times 3.0 \mu \mathrm{m}$ and images were collected at different position within the channel in order to reduce radiation damage. All diffraction intensity was normalized to the incident beam intensity. GIXRD measurements were performed using an incident angle chosen as being slightly larger than the critical angle for total reflection of the organic film $\left(\mathrm{ca} .0 .17^{\circ}\right)$ in order to penetrate through the full film depth. 
Electrical characterization: transfer curves were performed before and after the application of $\mathrm{V}_{\mathrm{G}}$ and $V_{\mathrm{DS}}$ in order to not perturb the film microstructure.

Computational simulations: DFT simulations of the tetramer were performed at level M06-2X/6$31 \mathrm{G}^{* 41}$ using the G09 software suite ${ }^{42}$. Geometry optimizaion and molecular dynamics simulations of the thin film were performed using a modified version of the TINKER software suite ${ }^{43,44}$, with $\mathrm{MM}^{45-47}$ force field and charges derived from the charge equilibration $(\mathrm{QEq})^{48}$ scheme. The implicit surface was modelled using a Lennard-Jones 6-3 potential. The gate bias was implemented for each atom as a z-dependent potential multiplied by the QEq charge.

\section{ACKNOWLEGMENTS}

The authors acknowledge A. Shehu for his collaboration at the early stage of the project, F. Borgatti for the fruitful discussion, J. R. Plaisier, G. Zerauschek and A. Lausi for optimizing the experimental set-up at the MCX-ELETTRA beamline, and V.L.R. Jacques and P. Evans for technical support at the ID01-ESRF beamline. Financial support for this research by the national Project N-CHEM, Flagship NANOMAX, by the DGI (Spain) with project BE-WELL CTQ201340480-R, Generalitat de Catalunya (2014-SGR-17) and Networking Research Center on Bioengineering, Biomaterials and Nanomedicine (CIBER-BBN).

\section{Supporting Information Available:}

X-ray damage study, details of X-ray diffraction measurements and theoretical calculation, and leakage effect are reported. This material is available free of charge via the Internet at http://pubs.acs.org.

\section{REFERENCES}

1. Dinelli, F.; Murgia, M.; Levy, P.; Cavallini, M.; Biscarini, F.; de Leeuw, D. M., Spatially correlated charge transport in organic thin film transistors. Physical Review Letters 2004, 92, (11), 116802.

2. Liscio, F.; Albonetti, C.; Broch, K.; Shehu, A.; Quiroga, S. D.; Ferlauto, L.; Frank, C.; Kowarik, S.; Nervo, R.; Gerlach, A.; Milita, S.; Schreibers, F.; Biscarini, F., Molecular Reorganization in Organic Field-Effect Transistors and Its Effect on Two-Dimensional Charge Transport Pathways. Acs Nano 2013, 7, (2), 1257-1264.

3. Shehu, A.; Quiroga, S. D.; D'Angelo, P.; Albonetti, C.; Borgatti, F.; Murgia, M.; Scorzoni, A.; Stoliar, P.; Biscarini, F., Layered Distribution of Charge Carriers in Organic Thin Film Transistors. Physical Review Letters 2010, 104, (24), 246602. 
4. Mas-Torrent, M.; Rovira, C., Role of Molecular Order and Solid-State Structure in Organic Field-Effect Transistors. Chemical Reviews 2011, 111, (8), 4833-4856.

5. $\quad$ Pfattner, R.; Mas-Torrent, M.; Bilotti, I.; Brillante, A.; Milita, S.; Liscio, F.; Biscarini, F.; Marszalek, T.; Ulanski, J.; Nosal, A.; Gazicki-Lipman, M.; Leufgen, M.; Schmidt, G.; Molenkamp, L. W.; Laukhin, V.; Veciana, J.; Rovira, C., High-Performance Single Crystal Organic Field-Effect Transistors Based on Two Dithiophene-Tetrathiafulvalene (DT-TTF) Polymorphs. Advanced Materials 2010, 22, (37), 4198-4203.

6. Lee, W. H.; Cho, J. H.; Cho, K., Control of mesoscale and nanoscale ordering of organic semiconductors at the gate dielectric/semiconductor interface for organic transistors. Journal of Materials Chemistry 2010, 20, (13), 2549-2561.

7. Woll, C., Physical and Chemical Aspects of Organic Electronics. Wiley-VHC: Weinheim, 2009.

8. Liscio, F.; Milita, S.; Albonetti, C.; D'Angelo, P.; Guagliardi, A.; Masciocchi, N.; Della Valle, R. G.; Venuti, E.; Brillante, A.; Biscarini, F., Structure and Morphology of PDI8-CN2 for nType Thin-Film Transistors. Advanced Functional Materials 2012, 22, (5), 943-953.

9. Mandal, T.; Garg, A.; Deepak, Thin film transistors fabricated by evaporating pentacene under electric field. Journal of Applied Physics 2013, 114, (15).

10. Schweicher, G.; Paquay, N.; Amato, C.; Resel, R.; Koini, M.; Talvy, S.; Lemaur, V.; Cornil, J.; Geerts, Y.; Gbabode, G., Toward Single Crystal Thin Films of Terthiophene by Directional Crystallization Using a Thermal Gradient. Crystal Growth \& Design 2011, 11, (8), 3663-3672.

11. De Luca, G.; Treossi, E.; Liscio, A.; Mativetsky, J. M.; Scolaro, L. M.; Palermo, V.; Samori, P., Solvent vapour annealing of organic thin films: controlling the self-assembly of functional systems across multiple length scales. Journal of Materials Chemistry 2010, 20, (13), 2493-2498. 12. Gentili, D.; Valle, F.; Albonetti, C.; Liscio, F.; Cavallini, M., Self-Organization of Functional Materials in Confinement. Accounts of Chemical Research 2014, 47, (8), 2692-2699. 13. Himmelberger, S.; Dacuna, J.; Rivnay, J.; Jimison, L. H.; McCarthy-Ward, T.; Heeney, M.; McCulloch, I.; Toney, M. F.; Salleo, A., Effects of Confinement on Microstructure and Charge Transport in High Performance Semicrystalline Polymer Semiconductors. Advanced Functional Materials 2013, 23, (16), 2091-2098.

14. Kang, B.; Jang, M.; Chung, Y.; Kim, H.; Kwak, S. K.; Oh, J. H.; Cho, K., Enhancing 2D growth of organic semiconductor thin films with macroporous structures via a small-molecule heterointerface. Nature Communications 2014, 5.

15. Viani, L.; Risko, C.; Toney, M. F.; Breiby, D. W.; Bredas, J. L., Substrate-Induced Variations of Molecular Packing, Dynamics, and Intermolecular Electronic Couplings in Pentacene Monolayers on the Amorphous Silica Dielectric. Acs Nano 2014, 8, (1), 690-700.

16. Giri, G.; Verploegen, E.; Mannsfeld, S. C. B.; Atahan-Evrenk, S.; Kim, D. H.; Lee, S. Y.; Becerril, H. A.; Aspuru-Guzik, A.; Toney, M. F.; Bao, Z. A., Tuning charge transport in solutionsheared organic semiconductors using lattice strain. Nature 2011, 480, (7378), 504-U124.

17. Ferlauto, L.; Liscio, F.; Orgiu, E.; Masciocchi, N.; Guagliardi, A.; Biscarini, F.; Samori, P.; Milita, S., Enhancing the Charge Transport in Solution-Processed Perylene Di-imide Transistors via Thermal Annealing of Metastable Disordered Films. Advanced Functional Materials 2014, 24, (35), 5503-5510.

18. Kowarik, S.; Gerlach, A.; Sellner, S.; Schreiber, F.; Cavalcanti, L.; Konovalov, O., Realtime observation of structural and orientational transitions during growth of organic thin films. Physical Review Letters 2006, 96, (12), 125504.

19. Moulin, J. F.; Dinelli, F.; Massi, M.; Albonetti, C.; Kshirsagar, R.; Biscarini, F., In situ Xray synchrotron study of organic semiconductor ultra-thin films growth. Nuclear Instruments \& Methods in Physics Research Section B-Beam Interactions with Materials and Atoms 2006, 246, (1), 122-126.

20. Dinelli, F.; Albonetti, C.; Kolosov, O. V., Ultrasonic force microscopy: Detection and imaging of ultra-thin molecular domains. Ultramicroscopy 2011, 111, (4), 267-272. 
21. Li, R. P.; Ward, J. W.; Smilgies, D. M.; Payne, M. M.; Anthony, J. E.; Jurchescu, O. D.; Amassian, A., Direct Structural Mapping of Organic Field-Effect Transistors Reveals Bottlenecks to Carrier Transport. Advanced Materials 2012, 24, (41), 5553-5558.

22. Horowitz, G., Organic field-effect transistors. Advanced Materials 1998, 10, (5), 365-377.

23. Nakamura, M.; Ohguri, H.; Goto, N.; Tomii, H.; Xu, M. S.; Miyamoto, T.; Matsubara, R.; Ohashi, N.; Sakai, M.; Kudo, K., Extrinsic limiting factors of carrier transport in organic field-effect transistors. Applied Physics a-Materials Science \& Processing 2009, 95, (1), 73-80.

24. Annibale, P.; Albonetti, C.; Stoliar, P.; Biscarini, F., High-resolution mapping of the electrostatic potential in organic thin-film transistors by phase electrostatic force microscopy. Journal of Physical Chemistry A 2007, 111, (49), 12854-12858.

25. Rivnay, J.; Jimison, L. H.; Northrup, J. E.; Toney, M. F.; Noriega, R.; Lu, S. F.; Marks, T. J.; Facchetti, A.; Salleo, A., Large modulation of carrier transport by grain-boundary molecular packing and microstructure in organic thin films. Nature Materials 2009, 8, (12), 952-958.

26. Gentili, D.; Di Maria, F.; Liscio, F.; Ferlauto, L.; Leonardi, F.; Maini, L.; Gazzano, M.; Milita, S.; Barbarella, G.; Cavallini, M., Targeting ordered oligothiophene fibers with enhanced functional properties by interplay of self-assembly and wet lithography. Journal of Materials Chemistry 2012, 22, (39), 20852-20856.

27. Sharma, A.; Mathijssen, S. G. J.; Kemerink, M.; M., d. L. D.; A., B. P., Proton migration mechanism for the instability of organic field-effect transistors Applied Physics Letters 2009, 95, (25), 253305

28. Eggeman, A. S.; Illig, S.; Troisi, A.; Sirringhaus, H.; Midgley, P. A., Measurement of molecular motion in organic semiconductors by thermal diffuse electron scattering. Nature Materials 2013, 12, (11), 1044-1048.

29. Chang, J. F.; Sakanoue, T.; Olivier, Y.; Uemura, T.; Dufourg-Madec, M. B.; G. Yeates, S.; Cornil, J.; Takeya, J.; Troisi, A.; Sirringhaus, H., Hall-effect measurements probing the degree of charge-carrier delocalization in solution-processed crystalline molecular semiconductors. Physical Review Letters 2011, 107, 066601

30. Cheng, H. L.; Chou, W. Y.; Kuo, C. W.; Wang, Y. W.; Mai, Y. S.; Tang, F. C.; Chu, S. W., Influence of electric field on microstructures of pentacene thin films in field-effect transistors. Advanced Functional Materials 2008, 18, (2), 285-293.

31. Juers, D. H.; Weik, M., Similarities and differences in radiation damage at $100 \mathrm{~K}$ versus 160 $\mathrm{K}$ in a crystal of thermolysin. Journal of Synchrotron Radiation 2011, 18, 329-337.

32. O'Neill, P.; Stevens, D. L.; Garman, E. F., Physical and chemical considerations of damage induced in protein crystals by synchrotron radiation: a radiation chemical perspective. Journal of Synchrotron Radiation 2002, 9, 329-332.

33. Neuhold, A.; Novak, J.; Flesch, H. G.; Moser, A.; Djuric, T.; Grodd, L.; Grigorian, S.; Pietsch, U.; Resel, R., X-ray radiation damage of organic semiconductor thin films during grazing incidence diffraction experiments. Nuclear Instruments \& Methods in Physics Research Section BBeam Interactions with Materials and Atoms 2012, 284, 64-68.

34. Yoneda, Y., Anomalous Surface Reflection of X Rays. Physical Review 1963, 131, 20102013.

35. Vineyard, G. H., Grazing-Incidence Diffraction and the Distorted-Wave Approximation for the Study of Surfaces. Physical Review B 1982, 26, (8), 4146-4159.

36. Warren, B. E., X-ray diffraction. Dover: New York, 1990.

37. Smilgies, D. M., Scherrer grain-size analysis adapted to grazing-incidence scattering with area detectors. Journal of Applied Crystallography 2009, 42, 1030-1034.

38. Schiefer, S.; Huth, M.; Dobrinevski, A.; Nickel, B., Determination of the crystal structure of substrate-induced pentacene polymorphs in fiber structured thin films. Journal of the American Chemical Society 2007, 129, (34), 10316-+. 
39. Brillante, A.; Bilotti, I.; Della Valle, R. G.; Venuti, E.; Girlando, A.; Masino, M.; Liscio, F.; Milita, S.; Albonetti, C.; D'angelo, P.; Shehu, A.; Biscarini, F., Structure and dynamics of pentacene on $\mathrm{SiO} 2$ : From monolayer to bulk structure. Physical Review B 2012, 85, (19).

40. Verlaak, S.; Heremans, P., Molecular microelectrostatic view on electronic states near pentacene grain boundaries. Physical Review B 2007, 75, (11).

41. Zhao, Y.; Truhlar, D. G., Density functionals with broad applicability in chemistry. Accounts of Chemical Research 2008, 41, (2), 157-167.

42. http://www.gaussian.com/index.htm.

43. TINKER Software Tools for Molecular Design. http://dasher.wustl.edu/tinker

44. Sandig, N.; Zerbetto, F., Molecules on gold. Chemical Communications 2010, 46, (5), 667676.

45. Allinger, N. L.; Yuh, Y. H.; Lii, J. H., Molecular Mechanics - the Mm3 Force-Field for Hydrocarbons .1. Journal of the American Chemical Society 1989, 111, (23), 8551-8566.

46. Lii, J. H.; Allinger, N. L., Molecular Mechanics - the Mm3 Force-Field for Hydrocarbons .2. Vibrational Frequencies and Thermodynamics. Journal of the American Chemical Society 1989, $111,(23), 8566-8575$.

47. Lii, J. H.; Allinger, N. L., Molecular Mechanics - the Mm3 Force-Field for Hydrocarbons .3. The Vanderwaals Potentials and Crystal Data for Aliphatic and Aromatic-Hydrocarbons. Journal of the American Chemical Society 1989, 111, (23), 8576-8582.

48. Rappe, A. K.; Goddard, W. A., Charge Equilibration for Molecular-Dynamics Simulations. Journal of Physical Chemistry 1991, 95, (8), 3358-3363. 